\title{
Primary Central Nervous System Hodgkin Lymphoma versus Lymphoproliferative Disorder in an Asymptomatic Immunocompromised Patient - A Case Report and Review of the Current Literature \\ Godbe $\mathrm{KN}^{1}$, Guilliams $\mathrm{EL}^{2}$, Benko $\mathrm{MJ}^{2}$, Grider $\mathrm{DJ}^{3}$ and Stump $\mathrm{MS}^{2 *}$
}

${ }^{1}$ VTCSOM, Virginia, USA

${ }^{2}$ Carilion Roanoke Memorial Hospital, Virginia, USA

${ }^{3}$ Dominion Pathology Associates, Virginia, USA

\begin{abstract}
Primary central nervous system (CNS) Hodgkin's lymphoma is extremely rare. An 82 year old female presented to the Emergency Department after she tripped and hit her head, resulting in a head laceration with no other apparent injuries. Head CT revealed a hyperdense area consistent with either a parieto-occipital hemorrhage or mass. She was transferred to a tertiary care institution, where head MRI revealed a parieto-occipital mass concerning for metastatic disease. No primary source or evidence of metastatic disease were found. The tumor was resected and identified to be a classical Hodgkin's lymphoma, mixed cellularity type. The patient had no complications and was discharged home. Recommendations for patient's future treatment included a PET scan and radiotherapy. After a consultation with a lymphoma expert at the $\mathrm{NIH}$, the patient's diagnosis was changed to an atypical lymphoproliferative disorder with Reed-Sternberg/Hodgkin like cells based on the patient's history of rheumatoid arthritis treated with an immunosuppressant. A review of the literature revealed several primary CNS Hodgkin's lymphoma cases that were extremely similar to the patient in this study. This case's diagnosis as an atypical lymphoproliferative disorder casts doubt on if previously reported cases of CNS primary Hodgkin's disease were misclassified as the pathological staining of this patient and the immunosuppressant history are extremely similar.
\end{abstract}

Keywords: Primary CNS tumor; Lymphoma; Hodgkin; Lymphoproliferative disorder

Abbreviations: CNS: Central Nervous System; HL: Hodgkin Lymphoma; LPD: Lymphoproliferative Disorder, EBV: Epstein Barr Virus

\section{Introduction}

Hodgkin Lymphoma (HL) is a systemic disease typically involving the lymph nodes. When systemic Hodgkin disease is present, the incidence of central nervous system (CNS) involvement is less than $0.2 \%$ (and has been reported as low as $0.02 \%$ ) [1,2]. Most of the cases with intracranial involvement of Hodgkin's disease present at relapse, with several at initial presentation as part of systemic disease. Intracranial HL very rarely presents as a primary lesion $[1,2]$. As of January 2019, there have only been 23 cases of primary CNS HL reported in the literature $[3,4]$. Common presentations of CNS involvement of Hodgkin's includes cranial nerve palsies, motor and/or sensory deficits, headaches, papilledema, coma, and seizures $[5,6]$. In this case report, we describe a patient diagnosed initially with isolated primary CNS HL. After consultation with the NIH, the diagnosis was changed to an atypical lymphoproliferative disorder (LPD) with ReedSternberg/Hodgkin like cells.

Risk factors for HL include previous infection by Epstein Barr virus (EBV) and immunosuppression, although the full underlying etiology is not yet understood [7]. The incidence of HL increases in patients with a history of autoimmune conditions, such as rheumatoid arthritis and systemic lupus erythematosus [7]. For LPDs, risk factors include immunosuppressant use, length of use, underlying disease type, infection by Epstein-Barr virus (EBV), and a patient's genetic predisposition [8]. The immunosuppressants most associated with increasing the risk of a lymphoproliferative disorder are methotrexate and azathioprine (although patient's using azathioprine are at less risk than those using methotrexate) [8]. The risk of the medication hydroxychloroquine on LPDs was not found in the literature at this time.
Classical HL has Reed-Sternberg cells in a reactive inflammatory background with CD 30(+), CD15(+), and CD45/LCA(-) [8]. Comparatively, Hodgkin-like LPDs have large cells that are CD20(+), CD30(+), CD45/LCA(+), and CD15(-) [8]. It is difficult to differentiate HL from HL LPDs due to the similar aforementioned risk factors and staining, especially if the LPD is CD15(+).

\section{Case Report}

An 82-year-old female with a history of HTN, hyperlipidemia, rheumatoid arthritis, chronic kidney disease, and an unknown type of skin cancer was admitted to a rural hospital after she sustained a head injury. She has no family history of cancer. Patient was treated for her rheumatoid arthritis with Plaquenil, but has been off of this medication for two years. The patient stated she slipped on a tree root while walking up a bank and fell forward, striking her head on a root. Patient did not lose consciousness and proceeded to the Emergency Department at a local hospital where she was treated for a head laceration and underwent head CT. The CT showed a hyper-dense parieto-occipital lesion, either hemorrhage or mass. The patient was then transferred to a tertiary care institution. Upon arrival, no other injuries were identified on physical exam and the patient denied neck pain, nausea, and vomiting although she did admit to intermittent headaches for the past few months.

*Corresponding author: Stump MS, Carilion Roanoke Memorial Hospital, Virginia, USA, Tel: 317-965-5514; E-mail: msstump@carilionclinic.org

Received August 08, 2019; Accepted August 23, 2019; Published August 28, 2019

Citation: Godbe KN, Guilliams EL, Benko MJ, Grider DJ, Stump MS (2019) Primary Central Nervous System Hodgkin Lymphoma versus Lymphoproliferative Disorder in an Asymptomatic Immunocompromised Patient - A Case Report and Review of the Current Literature. J Blood Lymph 9: 250.

Copyright: ( 2019 Godbe KN, et al. This is an open-access article distributed under the terms of the Creative Commons Attribution License, which permits unrestricted use, distribution, and reproduction in any medium, provided the original author and source are credited. 
Citation: Godbe KN, Guilliams EL, Benko MJ, Grider DJ, Stump MS (2019) Primary Central Nervous System Hodgkin Lymphoma versus Lymphoproliferative Disorder in an Asymptomatic Immunocompromised Patient - A Case Report and Review of the Current Literature. J Blood Lymph 9: 250.

A repeat CT brain without contrast showed a small focal hyperdensity in the left parieto-occipital region with surrounding edema (Figure 1). There was no midline shift. MRI was recommended to further evaluate for an underlying mass legion. The MRI showed an enhancing lesion on the inferior aspect of the left parietal lobe measuring $1.4 \mathrm{~cm}$ with adjacent white matter edema but without significant local masseffect (Figure 2). No other lesions were identified, but the findings were worrisome for underlying metastatic disease in a patient of her age group. A CT chest, abdomen, pelvis found no primary sources for the brain lesion, and no evidence of metastatic disease (Figure 3) [9-15].

The neurosurgery team started the patient on Decadron and Keppra. The patient remained stable without neurologic defects over the next few days.

On hospital day 5, she underwent a left parieto-occipital craniotomy for tumor resection Patient had $75 \mathrm{cc}$ of blood loss and was stable post procedure. Patient was AAOx4 with 5/5 motor strength and sensation intact. Her pain was controlled with prn acetaminophen, oxycodone, and fentanyl. Her blood pressure was 176/57 with an elevated BUN and creatinine due to her other medical conditions. Other laboratory findings were within normal limits [16-26].

On day 6, the patient was recovering well with stable lab values other than a low Hemoglobin and an increase in WBC count thought to be stress/steroid induced leukocytosis. Physical exam was within normal limits, and patient started physical therapy. The patient started occupational therapy on day 7. Patient functioned near baseline with physical components of mobility and activities of daily living but below

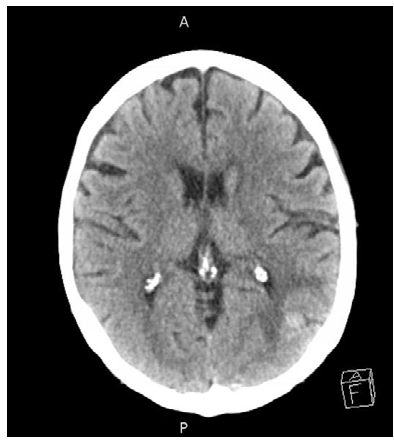

Figure 1: CT head/brain angled axial view with small focal hyper-density in left parieto-occipital region.
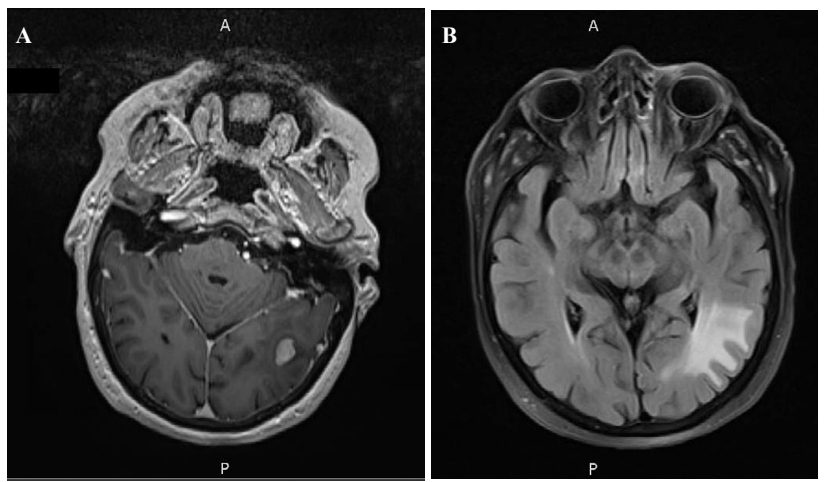

Figure 2: MRI brain w/wo contrast. Enhancing lesion on inferior aspect of left parietal lobe with adjacent white matter edema but no significant local masseffect. (A) Axial T1 Post Contrast (B) T2 Flare.

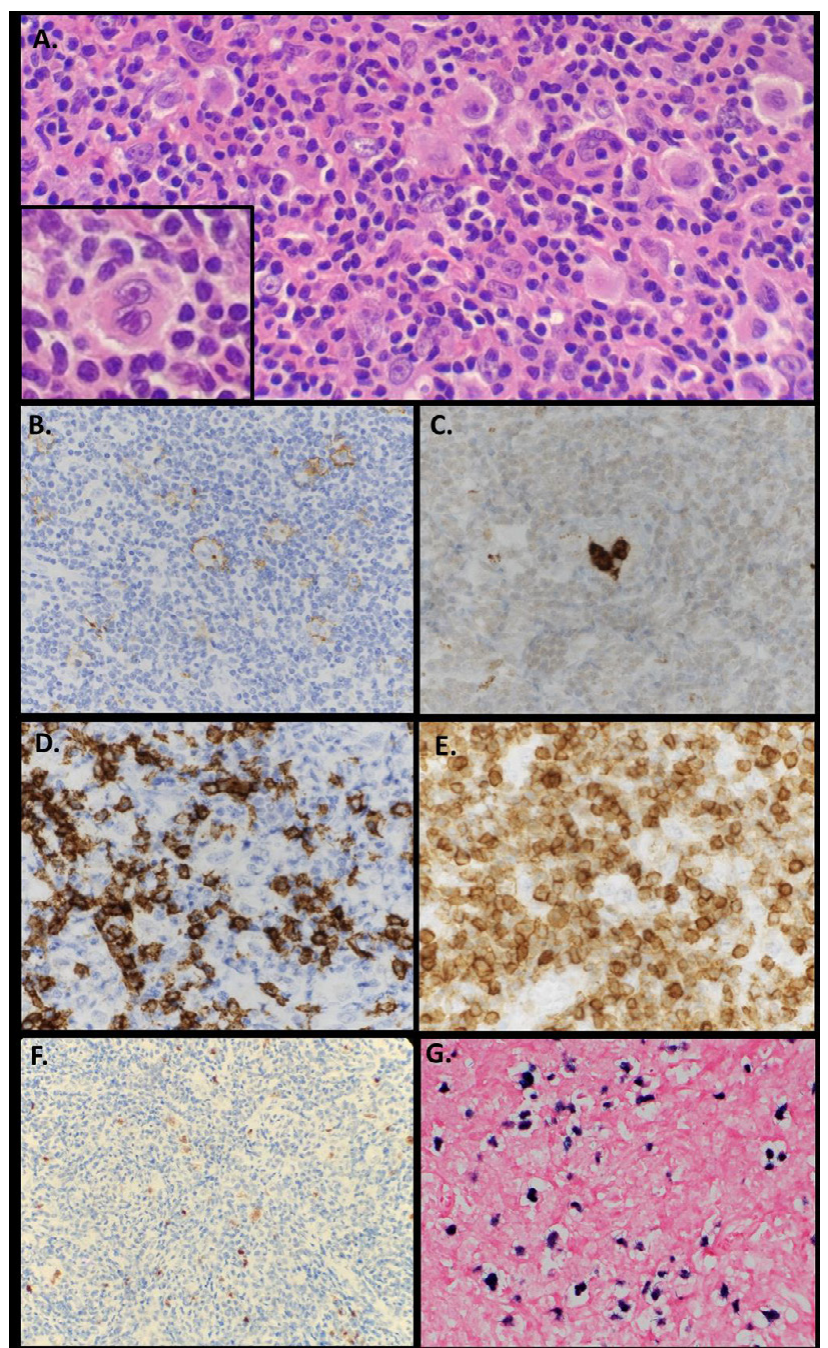

Figure 3: (A) H\&E stain, inset with Reed Sternberg cell. (B) Large cells stained for CD30. (C) CD15 positive. (D) CD20 negative in large cells (E) CD45 negative in large cells. (F) Pax5 dim nuclear staining. (G) EBV by ISH positive. Images were acquired using an Olympus BX41 microscope and Pixel 2XL camera.

baseline with vision, memory, and cognitive components of activities of daily living.

The pathology report originally indicated the left occipital brain mass to be a classical Hodgkin's lymphoma, mixed cellularity subtype. Scattered small clusters of large atypical cells with abundant slightly eosinophilic cytoplasm and regular nuclei were present, some with multiple nuclei and prominent nucleoli consistent with ReedSternberg cells (CD30 positive in large cells, CD45 negative in large cells). A consult with a lymphoma expert NIH disagreed with this interpretation and the diagnosis was changed to EBV-positive atypical lymphoproliferative disorder with Reed-Sternberg/Hodgkin like cells.

As patient was stable (other than relatively poor blood pressure control), discharge planning was initiated. Patient was discharged on post-operative day 3 (day 9 of hospital stay). Recommendations for patient's future treatment included a PET scan and radiation treatment (36 Gy). The patient is now being followed by Med/Onc. 
Citation: Godbe KN, Guilliams EL, Benko MJ, Grider DJ, Stump MS (2019) Primary Central Nervous System Hodgkin Lymphoma versus Lymphoproliferative Disorder in an Asymptomatic Immunocompromised Patient - A Case Report and Review of the Current Literature. J Blood Lymph 9: 250.

\section{Discussion}

The main differentials for this patient included an intracerebral hemorrhage (due to patient's fall), or a metastatic lesion due to her demographic. HL was not originally on the differential list as, in addition to a primary CNS lesion being uncommon, the patient is older than the reported age of other patients with Primary CNS HL (average age at onset is 56 years with a range of 34-84 years [4]) and lacks the common presentation of cranial nerve palsies or deficits [1]. Once this rare diagnosis was made, the specimens were sent to the NIH for a second opinion. A lymphoma expert at the NIH disagreed with the original diagnosis based on the patient's history of immunosuppression and EBV positivity. The diagnosis was changed to an EBV-positive B-cell lymphoproliferative disorder with Hodgkin/ Reed-Sternberg-like cells.

A review of the literature found 23 cases of primary CNS HL. The cases are tabulated in Figure 4. Interestingly, the patient in case 21 was treated with methotrexate while the patients in case 19 and 20 were both treated with azathioprine (medications strongly associated with LPDs). Additionally, cases 19 and 20 were EBV positive (not investigated in case 21 ). Case 19, 20, and 21 had similar staining to the patient discussed in this report. Based on their history and staining profile, these three cases may actually represent HL-like LPDs. The similar risk factors and stains cast doubt on how to make a true diagnosis of primary CNS Hodgkin's lymphoma.

Assuming that all currently reported cases of primary CNS HL are indeed HL rather than aPD, there are more cases in males (15) than in females (8). Of the 23 cases, $60.9 \%$ (14) are immunocompetent, $26.1 \%$ (6) have an unknown immune status, while $13.0 \%$ (3) are immunocompromised. These are the same three cases that may be LPDs rather than primary HL. No patient has a history of HIV. A 2008 study found that $37.5 \%$ of patients with systemic HL including the CNS $(n=16)$ were immunocompromised [1]. This is greater than the
$13.0 \%$ of immunocompromised patients making up all reported cases of primary CNS HL. Perhaps the link between immunodeficiency is not as strong between systemic HL and primary CNS HL. Or perhaps when the patient is immunocompromised in the case of a CNS lesion the disease is classified as HL-like LPD.

Out of the current count of primary CNS HL cases $72 \%$ of cases were EBV positive (eight cases were positive, three that are negative, and twelve cases that were not investigated). A study examining the EBV association in systemic HL with CNS involvement found only $44 \%$ of cases were EBV positive. This is another interesting difference between primary CNS HL and systemic HL with CNS involvement. It is important to note that the sample size in these comparisons are low due to the rarity of both presentations. It is also important to note that LPDs are also strongly associated with EBV positivity.

Of the four main subtypes of Hodgkin's lymphoma, only nodular sclerosing and mixed cellularity have been reported as primary CNS lesions. Five cases are mixed cellularity, while seven are nodular sclerosing with the rest remaining unspecified.

Primary CNS HL has a good prognosis, with no evidence of recurrence as long as ten years after initial diagnosis. The LPDs similarly have good prognosis - spontaneous regression of the lesion in response to drug withdrawal has been recorded in most EBV positive cases [27]. The patient discussed in this report was already off of her immunosuppressive therapy for two years when her diagnosis was made. Treatment for CNS HL typically consists of radiation therapy with 30-45 Gy after resection (if possible), as was recommended for the case discussed in this report. Patients have also been treated successfully with chemotherapy and combination therapy in the past. This report casts doubt upon need for radiation and chemotherapy was indeed necessary for cases 19, 20, and 21 if the diagnosis was a HL-like LPD.

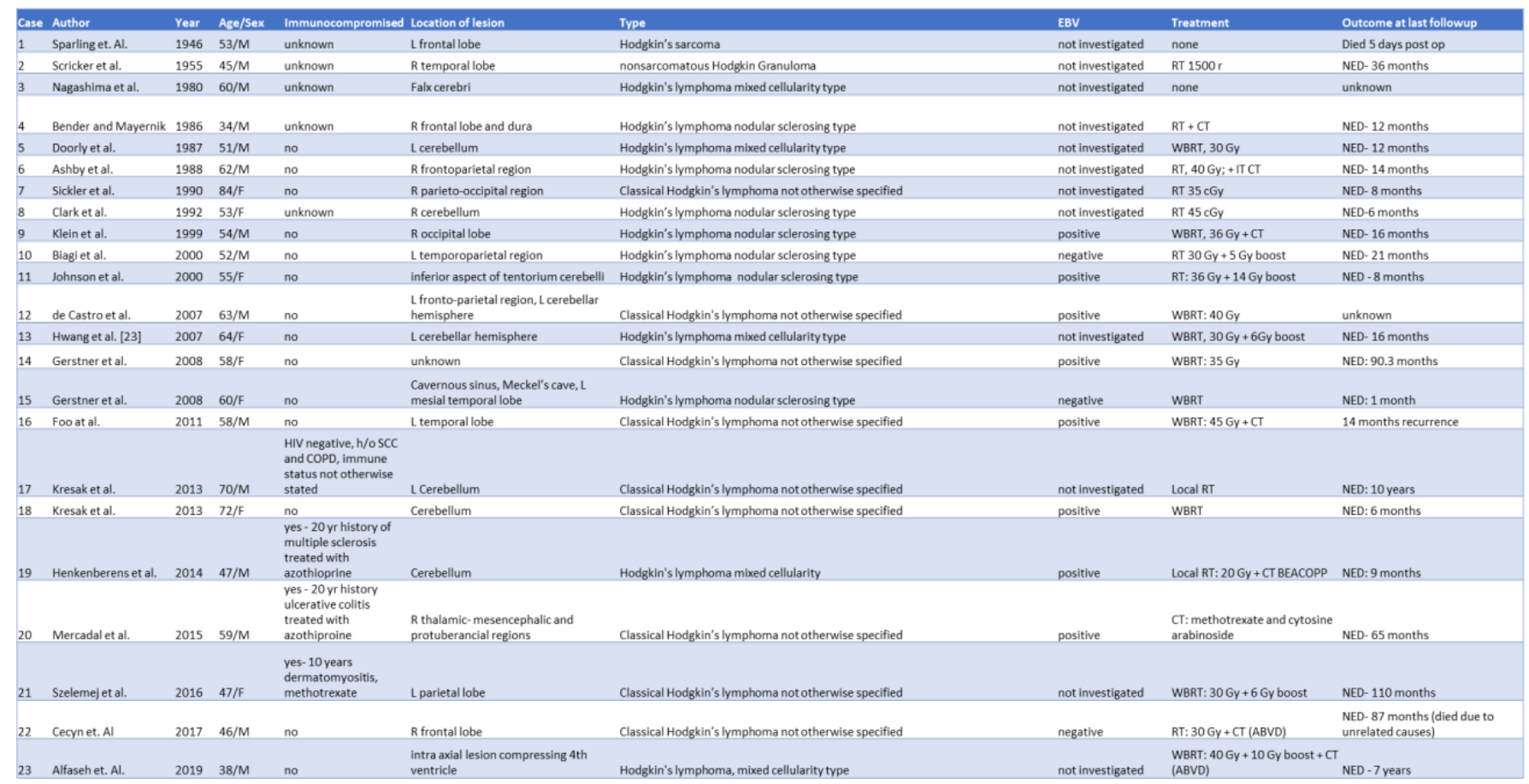

Figure 4: Review of current literature of primary CNS Hodgkin lymphoma patients [1,3,4,9-26] 
Citation: Godbe KN, Guilliams EL, Benko MJ, Grider DJ, Stump MS (2019) Primary Central Nervous System Hodgkin Lymphoma versus Lymphoproliferative Disorder in an Asymptomatic Immunocompromised Patient - A Case Report and Review of the Current Literature. J Blood Lymph 9: 250.

\section{Conclusion}

This case report and literature review addresses the ambiguity of diagnosing primary HL versus HL-like LPD. Both of these diagnoses have similar risk factors and similar good prognoses. The basis of diagnosis based the patient's medical history when pathological staining appears to fit both HL and HL-like LPD leads to question what guidelines might be created next to better differentiate these two disease processes. By better differentiating these two processes, we can aim to prevent unnecessary radiation to patients with HL-like LPD.

\section{References}

1. Gerstner ER, Abrey LE, Schiff D, Ferreri AJM, Lister A, et al. (2008) CNS Hodgkin lymphoma. Blood 112: 1658-1661.

2. Morawa E, Ragam A, Sirota R, Nabhan C (2007) Hodgkin's lymphoma involving the CNS. J Clin Oncol 25: 1437-1438.

3. Alfaseh A, Rajeh MN, Hamed G (2019) Primary central nervous system Hodgkin Lymphoma: A case discussion and a hypothesis on the etiology. Avicenna J Med 9: 28-31.

4. KZ C, EM C, JS O (2017) Primary central nervous system involvement in classical Hodgkin's lymphoma: Case report and review of the literature. J Blood Lymph 8:196.

5. van Blydenstein SA, Patel M, Philip V, Lakha A, Pather S, et al. (2014) Classical Hodgkin Lymphoma involving the central nervous system (brain) - an unusual presentation. Clin Case Rep 2: 88-92.

6. Akyüz C, Yalcin B, Atahan IL, Varan A, Kutluk MT, et al. (2005) Intracranial Involvement in Hodgkin's Disease. Pediatr Hematol Oncol 22: 589-596.

7. Shanbhag S, Ambinder RF (2018) Hodgkin lymphoma: A review and update on recent progress. CA Cancer J Clin 68: 116-132.

8. Medeiros LJ, Muzzafar T, Miranda RN (2019) Immunomodulating AgentAssociated Lymphoproliferative Disorders. 2019. In: Lymph Nodes and Extranodal Lymphomas.

9. Bender BL, Mayernik DG (1986) Hodgkin's Disease Presenting With Isolated Craniospinal Involvement. Cancer 58:1745-1748.

10. Mercadal S, Climent F, García N, González-Barca E (2015) Long Term Remission of a Primary Intracerebral Hodgkin Lymphoma in a Patient Previously Treated With Azathioprine. J Integr Oncol 4: 144.

11. Hwang CY, Song YJ, Kim DC, Choi SS, Choi YM, et al. (2007) Primary Cerebellar Hodgkin's Lymphoma. J Korean Neurosurg 42:149-152.

12. Henkenberens C, Franzke A, Raab P, Oschlies I, Klapper W, et al. (2014) Primary EBV-positive Hodgkin's lymphoma of the CNS under azathioprine treatment: case report and review of the literature. Strahlenther Onkol 190: 847-852.

13. Kresak JL, Nguyen J, Wong K, Davis R (2013) Primary Hodgkin lymphoma of the central nervous system: two case reports and review of the literature. Neuropathology 33: 658-662.

14. Biagi J, MacKenzie RG, Lim MS, Sapp M, Berinstein N (2000) Primary Hodgkin's disease of the CNS in an immunocompetent patient: A case study and review of the literature. Neuro-Oncology 2000:239-243.

15. Nagashima K, Mori S, Yoshimasu N, Takahashi K (1980) Primary Hodgkin's Disease of the Falx Cerebri. Acta Neuropathol 51: 161-163.

16. de Castro AF, Junior AS, de Lins e Horta H, Neuenschwander LC, Fonseca RP, et al. (2007) Primary intracerebral Hodgkin lymphoma. Br J Haematol 138 562 .

17. Schricker JL Jr, Smith DE (1955) Primary intracerebral Hodgkin's disease. Cancer 8: 629-633.

18. Johnson MD, Kinney MC, Scheithauer BW, Briley RJ, Hamilton K, et al. (2000) Primary intracerebral Hodgkin's disease mimicking meningioma: case report. Neurosurgery 47: 454-456.

19. Klein R, Müllges W, Bendszus M, Woydt M, Kreipe H, et al. (1999) Primary Intracerebral Hodgkin's Disease: Report of a Case with Epstein-Barr Virus Association and Review of the Literature. T Am J Surg Pathol 23: 477-481.

20. Doorly TP, Farrell MA, Phillips J (1987) Primary intracerebral Hodgkin's lymphoma. Journal of Neurology, Neurosurgery and Psychiatry 50: 1048-1050.

21. Ashby MA, Barber PC, Holmes AE, Freer CEL, Collins RD (1988) Primary Intracranial Hodgkin's Disease. The American Journal of Surgical Pathology 12: $294-299$

22. Szelemej PA, Bigder MG, Krcek J (2017) Treatment and long-term follow-up of primary CNS classical Hodgkin's lymphoma - A case report and review of the literature. Interdisciplinary Neurosurgery 9: 30-33.

23. Sparling HJ Jr, Adams RD (1946) Primary Hodgkin's sarcoma of the brain. Arch Pathol 42: 338-344.

24. Sickler GK, Hanson SK, Hsu SM, Papasozomenos SC (1990) Primary intracerebral Hodgkin's disease: a case report. Clinical neuropathology 9:143147.

25. Clark WC, Callihan T, Schwartzberg L, Fontanesi J (1992) Primary intracrania Hodgkin's lymphoma without dural attachment. Journal of neurosurgery 76 : 692-695.

26. Foo WC, Desjardins A, Cummings TJ (2010) Primary intracerebral Hodgkin lymphoma with recurrence. clinical neuropathology 30: 75-79.

27. Luca DC (2019) Other iatrogenic immunodeficiency-associated lymphoproliferative disorders. Pathology Outlines.com 